Conclusion This quadruple 24 weeks regimen has excelled the RVR, EVR, ETVT over SOC with DAAs over $11 \%$, with SVR $67 \%$. Needs a larger trial for validation

Disclosure of Interest None Declared.

\section{PWE-130 EFFECT OF N ACETYLCYSTEINE (NAC) IN HYPOXIA INDUCED LIVER INJURY (HILI)-A RANDOMIZED PLACEBO CONTROL CLINICAL TRIAL}

doi:10.1136/gutjnl-2013-304907.418

1."P Basu, ${ }^{2} \mathrm{~N} J$ Shah, ${ }^{3} \mathrm{~S}$ Farhat, ${ }^{1} \mathrm{R}$ Siriki, ${ }^{1} \mathrm{~K}$ Mittimanj, ${ }^{1} \mathrm{M}$ Rahaman, ${ }^{1} \mathrm{~S}$ Atluri. ${ }^{1}$ Forest Hills Hospital, Hofstra North Shore-LIJ School of Medicine; '2James J Peters VA Medical Center, Mount Sinai School of Medicine, New York; ${ }^{3}$ Columbia School of Physicians and Surgeons, NY, New York, United States

Introduction HILI is common with a prevalence of $10 \%$ in US. Transient shift of intra hepatic hemodynamic compromise leads to tissue hypoxia and induces hypoxia induced protein (HIP), heat shock protein 70 (HSP24.70), Endothelial reticular stress (ER) leading to reperfusion injury (RI). Dramatic rise of transaminases, drastic reversal with restoration of perfusion in weeks follows. In cirrhotics HILI requires liver transplantation. This study evaluated spontaneous recovery and salvage in HILI utilising NAC.

Methods Sixty patients $(n=60)$ with mean arterial pressure $(\mathrm{MAP})<35 \%$ and normal LFTs at base line. Group A $(\mathrm{n}=28)$ chronic liver disease (CLD) [alcohol-11/28 (39\%), NASH-9/28 (32\%), Hepatitis C-4/28 (14\%), hepatitis B-2/28 (7\%), PBC-1/28 (3\%), AIH-1/28 $(3 \%)$. Group B $(\mathrm{n}=32)$ [respiratory failure-12/32 (37\%), CHF-8/32 (25\%), CVA-2/32 (6\%), sepsis-6/32 (19\%), post op-4/32 (12\%)]. Randomized into Placebo group- A1 (14) \& B1 (16) and IV NAC for 48 hours - A2 (14) \& B2 (16). Serum Transaminases, Bilirubin, INR, Creatinine and MELD score at $0,3^{\text {rd }}, 6^{\text {th }}$, 9th and 12 th days with MAP and modified Sequential Organ Failure Assessment (SOFA) Score. All patients were allowed standard of care (SOC) and resuscitations if needed.

Exclusions: Organ transplant, Septic shock, Hemodialysis, cancer, acute myocardial Infarct, Tylenol injury, acute viral hepatitis and organ trauma.

Results Placebo groups A1, B1: Normalized A1-[LFTs- on $3^{\text {rd }}$ day$(7 \%), 6^{\text {th }}$ day-(21\%), 9th day- $(36 \%)$ and 12 th day- $(21 \%) .1 / 14(7 \%)$ died]. B1(CLD) [ LFTs $3^{\text {rd }}$ day-(19\%) $6^{\text {th }}(44 \%) 9$ th $(25 \%), 2 / 16(6 \%)$ died of sepsis] NAC Groups A2[normalised LFTs 3rd (57\%) $6^{\text {th }}$ day(43\%) 9 th day (25\%), (7\%)-one died ] B2 (CLD) [Normalized LFTs$3^{\text {rd }}$ day-(63\%), $6^{\text {th }}$ day-(25\%) 9 th1/16(6\%), one died]

Conclusion This Study postulates that IV NAC (A2, B2) has efficient spontaneous recovery and salvage in non-CLD sub group $\mathrm{B} 2$ $(63 \%)>\mathrm{A} 2(57 \%)$ in day 3 , in CLD NAC (A2) > placebo (A1) clinical recovery over placebo at $3^{\text {rd }}$ day, $(44 \%)$ over $(36 \%)-6^{\text {th }}$ day. Larger trial need to establish the routine usage of IV NAC in HILI.

Disclosure of Interest None Declared.

\section{PWE-131 DOES CAPSULE ENDOSCOPY HAVE A ROLE IN PATIENTS WITH CHRONIC LIVER DISEASE AND OBSCURE GASTROINTESTINAL BLEEDING}

\section{doi:10.1136/gutjnl-2013-304907.419}

1."P S Sidhu, 'M E McAlindon, 'R Sidhu. 'Gastroenterology, Royal Hallamshire Hospital, Sheffield Teaching Hospitals, Sheffield, UK

Introduction Chronic liver disease (CLD) is commonly associated with anaemia. Whilst varices represent the commonest cause of gastrointestinal bleeding in patients with CLD, in patients where iron deficiency anaemia (IDA) persist, capsule endoscopy (CE) may have a useful role to investigate the small bowel (SB). We conducted a study to evaluate the utility of CE in patients with CLD and obscure gastrointestinal bleeding (OGB) and their subsequent management consequences.
Methods We retrospectively analysed our data set and isolated patients with OGB and CLD. Data collected included demographics, clinical indication (overt bleeding $(\mathrm{OB})$ or IDA) the presence of co-morbidity, diagnostic yield (DY) and subsequent follow up.

Results Of the 1324 patients investigated for OGB using CE, $3 \%(n=41)$ had CLD. The mean age was 61 years (range $=26-88)$ with $59 \%$ males. The indications for CE was IDA in $66 \%(n=27)$ and $\mathrm{OB}$ in the remaining $34 \%$. All patients in this cohort had other significant co-morbidity in addition to CLD. Five patients were on non-steroidal anti-inflammatories whilst 2 patients were transfusion dependent. The DY (as defined by lesions responsible for OGB) identified on CE was $49 \%(n=20)$. The commonest finding was $\mathrm{SB}$ ulcer and erosions $27 \%(\mathrm{n}=11)$ and $\mathrm{SB}$ angioectasia (AE) $24 \%(\mathrm{n}=10)$. Other findings included SB varices $(2)$, blood without definite source (5), a tumour (metastatic renal tumour) and other miscellaneous lesions (4). In 13 patients (32\%), lesions found were within the upper GI tract, which had been underestimated at the index gastroscopy. These included gastric antral vascular ectasia (3), varices (oesophageal and duodenal)(2), blood without definite source (5) and others lesions (erosions, ulcers, portal hypertension and polyps )(9). In 2 patients, colonic lesions were identified (erosions and AE). There was no significant difference in the DY between those with IDA and OB $(p=0.59)$ and between the sexes $(p=0.41)$. In our cohort, management was altered in $90 \%(n=18)$ of those with a DY, in the form of further procedures $(25 \%, n=5)$ which included repeat OGD (2), colonoscopy (2), double balloon enteroscopy (1) and the patients with renal metastasis avoided surgery. $25 \%(n=5)$ of patients within this cohort also received argon photocoagulation therapy. On logistic regression, factors that were associated with a subsequent change in management included previous transfusions $(p=0.04)$ and SB AE $(p=0.03)$.

Conclusion $\mathrm{CE}$ is a useful tool for investigation of OGB in patients with chronic liver disease and persistent anaemia. Ulcers and $\mathrm{AE}$ were the commonest pathology seen in the SB in patients with CLD, in keeping with the published literature. CE is also useful to pick up pathology in the upper GI tract which may have been underestimated.

Disclosure of Interest None Declared.

\section{PWE-132 THE PREVALENCE AND CLINICAL SIGNIFICANCE OF CRYOGLOBULINAEMIA IN A SCOTTISH COHORT OF HEPATITIS C PATIENTS}

doi:10.1136/gutjnl-2013-304907.420

1."R E Swann, 'M Robinson, ${ }^{2} \mathrm{~S}$ Barclay, ${ }^{2} \mathrm{M}$ Priest, ${ }^{2} \mathrm{P}$ Mills, ${ }^{1} \mathrm{~J}$ McLauchlan, ${ }^{1} \mathrm{~A}$ Patel. ${ }^{1} M R C$ University of Glasgow Centre for Virus Research, University of Glasgow; ${ }^{2}$ Gastroenterology, NHS Greater Glasgow and Clyde, Glasgow, UK

Introduction Hepatitis $\mathrm{C}$ virus (HCV) infection is the most common cause of cryoglobulinaemia - a clonal B cell disorder characterised by precipitation of antibody aggregates on serum cooling. This can lead to vasculitic symptoms and complications including renal failure [1]. One meta-analysis suggested a prevalence of $44 \%$ in HCV infected patients. However, other studies have reported much lower rates [2]. Genotype is thought to influence prevalence, and data from the UK, where $45 \%$ of cases are genotype 3 , is unknown. This study aimed to determine the prevalence of cryoglobulinaemia in a cohort of HCV infected patients and identify any associated clinical features.

Methods 75 patients with chronic G1 or G3 HCV were prospectively recruited from liver clinics in addition to 20 healthy controls. None had a prior diagnosis of cryoglobulinaemia. Each patient completed a symptom questionnaire and clinical and laboratory details were recorded. A whole blood sample was collected and maintained at $37^{\circ} \mathrm{C}$ until serum had been separated using a heated centrifuge. Serum was stored at $4^{\circ} \mathrm{C}$ for 7 days. A patient was recorded as 